\title{
Componentes de variância e parâmetros genéticos em uma população multirracial Nelore-Angus sob enfoque Bayesiano ${ }^{1}$
}

\author{
Mauricio Morgado de Oliveira ${ }^{2 *}$, Fernando Flores Cardoso ${ }^{3}$, José Carlos da Silveira Osório ${ }^{4}$ \\ 1 Pesquisa financiada pelo CNPq. \\ 2 Departamento de Zootecnia/FAEM, UFPEL, Pelotas, RS. Bolsista do CNPq. \\ ${ }^{3}$ Embrapa Pecuária Sul - Bagé, RS. Bolsista de Produtividade do CNPq. \\ ${ }^{4}$ Departamento de Zootecnia/FAEM, UFPEL, Pelotas, RS. Bolsista de Produtividade do CNPq
}

RESUMO - Os objetivos neste estudo foram utilizar um modelo animal multirracial para verificar a presença de heterogeneidade de variância genética entre as raças Angus e Nelore, avaliar a importância da variância devida à segregação entre essas duas raças e estimar os efeitos genéticos aditivos de raça, dominância e epistasia aditiva $\times$ aditiva. Foram utilizados neste estudo 4.016 registros de ganho de peso pós-desmame do rebanho de formação da raça Brangus-Ibagé da Embrapa Pecuária Sul, analisados por três modelos distintos: modelo animal multirracial com segregação, modelo animal multirracial sem segregação; e modelo animal tradicional. A heterose reduziu substancialmente em cruzamentos avançados. A variância genética média a posteriori da raça Angus $\left(189 \pm 26 \mathrm{~kg}^{2}\right)$ foi maior que o dobro da obtida para a raça Nelore $\left(82 \pm 23 \mathrm{~kg}^{2}\right)$ e a variância da segregação entre essas raças foi de $11 \pm 5 \mathrm{~kg}^{2}$. A alteração das estimativas das variâncias genéticas raciais foi insignificante quando a variância da segregação foi fixada em zero. Critérios bayesianos de escolha apontam o modelo multirracial sem segregação entre raças como uma alternativa parcimoniosa adequada para avaliação genética dessa população.

Palavras-chave: Angus, avaliação genética, inferência bayesiana, multirracial, Nelore

\section{Variance components and genetic parameters in a Nelore-Angus multibreed population under Bayesian Approach}

\begin{abstract}
The objectives of this study were to use a multibreed animal model to verify the presence of heterogeneity of genetic variance between Angus and Nelore breeds, to verify the importance of the variance due to the segregation between these two breeds and to estimate breed additive genetic effects on the dominance and additive $\times$ additive epistatic effects. It was used in this study 4,016 records of post weaning weight gain from Brangus breed foundation herd from Embrapa Pecuária Sul, analyzed by three different models: multibreed animal model with segregation, multibreed animal model without segregation and traditional animal model. Heterosis was substantially reduced in advanced crosses. The posterior mean genetic variance of the Angus breed $\left(189 \pm 26 \mathrm{~kg}^{2}\right)$ was more than twofold of that obtained for the Nelore breed $\left(82 \pm 23 \mathrm{~kg}^{2}\right)$ and segregation variance between these two breeds was $11 \pm 5 \mathrm{~kg}^{2}$. The change in the breed genetic variance estimates was not significant when the segregation variance was set to zero. Bayesian choice criteria point to the multibreed animal model without segregation between breeds as an adequate parsimonious alternative for genetic evaluation of this population.
\end{abstract}

Key Words: Angus, Bayesian inference, genetic evaluation, multibreed, Nelore

\section{Introdução}

O cruzamento entre raças com diferentes características produtivas e de adaptação é uma ferramenta valiosa para aumentar a eficiência dos sistemas de produção de carne por meio da retenção de heterose e da complementaridade (Gregory et al., 1999). Diversas raças compostas ou sintéticas foram formadas em diferentes partes do mundo com a finalidade de produzir genótipos adaptados às condições de ambiente, ao sistema de produção e ao mercado. O Brangus-Ibagé desenvolvido na Estação Experimental Cinco Cruzes de Bagé, hoje Embrapa Pecuária Sul, é um desses exemplos, formado para combinar as características de habilidade materna, precocidade e qualidade de carne da raça Angus com a fertilidade, rusticidade e o rendimento de carcaça da raça Nelore (Salomoni et al., 1984).

Além do cruzamento, a seleção é outro fator genético para melhorar a produção de bovinos de corte. A resposta à seleção é proporcional à precisão das predições do mérito 
genético (Falconer \& Mackay, 1996). Em populações multirraciais, especialmente quando as raças fundadoras e grupos genéticos básicos de formação estão presentes, além daqueles animais de geração avançada após a estabilização da heterose e composição racial, a precisão dessas predições depende da estimação fidedigna das médias específicas por composição racial, dos desvios dos animais em relação a estas médias e das covariâncias genéticas entre parentes puros e/ou cruzados (Fernando, 1999; Cardoso \& Tempelman, 2004). Desse modo, o mérito genético de um animal é compreendido pela média de seu grupo de composição racial mais seu desvio individual específico em relação ao grupo (Arnold et al., 1992; Elzo, 1994; Klei et al., 1996).

A fim de usar mais eficientemente os registros de desempenho dos animais para cálculo do mérito genético, é importante modelar de forma correta as médias genotípicas e as covariâncias genéticas entre parentes como especificadas por Lo et al. (1993), considerando a existência de variabilidade genética particular a cada grupo racial em um modelo de efeitos genéticos aditivos, que no caso de população multirracial diferem do padrão usado nas avaliações de raças puras.

Recentemente um modelo animal multirracial, que inclui efeitos genéticos fixos aditivos e não aditivos com desvios individuais aditivos aleatórios baseados em Lo et al. (1993), foi proposto como sendo uma alternativa satisfatória e parcimoniosa para a avaliação genética de populações compostas (Cardoso \& Tempelman, 2004).

Os objetivos desse estudo foram: utilizar um modelo animal multirracial para verificar a presença de heterogeneidade de variância genética nas raças Angus e Nelore em uma população obtida pelo cruzamento entre essas raças e avaliar a importância da variância devida à segregação decorrente do cruzamento entre essas duas raças; estimar coeficientes genéticos para os efeitos aditivos de raça, de dominância e epistasia aditiva $\times$ aditiva em uma população obtida pelo cruzamento entre as raças Angus e Nelore, e comparar os valores genéticos obtidos por meio do modelo animal tradicional e o modelo animal multirracial.

\section{Material e Métodos}

Foram utilizados registros de ganho pós-desmame padronizados para um período de teste de 345 dias, coletados no rebanho de formação da raça Brangus-Ibagé (3/8 Nelore $\times$ 5/8 Angus) da Embrapa Pecuária Sul, em Bagé/RS. Esses registros foram analisados para comparar diferentes pressuposições sobre a variância genética aditiva de uma população multirracial. A formação desse rebanho foi iniciada nos meados de 1940, mas dados de peso corporal foram registrados somente a partir de 1956, quando nasceu a primeira geração de animais 5/8 Angus + 3/8 Nelore, e perdurou até 1979.

Do total de 6.499 nascimentos controlados e 110.250 registros de peso corporal em diferentes idades, coletados entre 1956 e 1979, foram excluídos animais sem registro de peso ao desmame (restando 6.366). Para obter o ganho pósdesmame entre as múltiplas pesagens disponíveis, foram formados grupos de contemporâneos considerando o ano e a estação de nascimento, além de sexo e da data da pesagem; sendo mantidos aqueles em que a média de idade no grupo de contemporâneos estava mais próxima de 550 dias, sendo eliminados os grupos de contemporâneos com idade média, na data da pesagem, mais de 60 dias distante de 550 dias e com menos de quatro animais, restando 4.097 animais com registro de ganho pós-desmame. Foram ainda eliminados os registro com desvio-padrão maior ou menor que 3,5 em relação a média do grupo de contemporâneos, permanecendo 4.060 registros no arquivo de trabalho.

Finalmente, foi testada a conectabilidade entre grupos contemporâneos com base no número total de laços genéticos (mínimo 10), pelo programa AMC (Roso \& Schenkel, 2006), que eliminou 44 animais desconectados pertencentes a nove grupos contemporâneos, restando 4.016 registros a serem analisados. As composições raciais variaram de Angus puro a 3/4 Nelore, sem nenhum animal Nelore puro. Todos os animais foram criados em pastejo sob condições extensivas.

O modelo animal multirracial utilizado neste estudo é baseado no modelo hierárquico bayesiano proposto por Cardoso \& Tempelman (2004), que pressupõe variâncias genéticas específicas para cada raça e variância genética devida à segregação entre raças, que corresponde à variância adicional observada na geração $\mathrm{F}_{2}$ em relação à $\mathrm{F}_{1}$ (Lo et al., 1993). Foi adotado o seguinte modelo linear misto:

$$
y_{i j k l}=\mu+g c_{i}+\operatorname{sexo}_{j}+\beta_{I D V}\left(i d v_{k}\right)+\beta_{I D V^{2}}\left(i_{d v_{k}^{2}}^{2}\right)+\beta_{A}
$$
$\left(f_{l}\right)+\beta_{D}\left(\delta_{l}\right)+\beta_{A A}\left(2\left[1-f_{l}\right] f_{l}\right)+u_{l}+e_{i j k l}$ em que: $\mathrm{y}_{\mathrm{ijkl}}=$ registro de GPD no l-ésimo animal, do i-ésimo GC e j-ésimo sexo; $\mathrm{m}$ = constante; $\mathrm{gc}_{\mathrm{i}}=$ efeito do i-ésimo GC (i $=1,2, \ldots, 268)$, com gc $_{\mathrm{i}} \sim N\left(0, \sigma_{g c}^{2}\right)$ para todo $\mathrm{i} ; \mathrm{sexo}_{\mathrm{j}}=$ efeito do j-ésimo sexo $(j=1, j=2$ e $j=3$ para macho, macho castrado e fêmea, respectivamente); $\beta^{*}$ 's = coeficientes de regressão associados com idade da vaca $\mathrm{k}$ mãe do l-ésimo animal, em meses $\left(\operatorname{IDV}_{\mathrm{k}}, 23 \leq \mathrm{IDV}_{\mathrm{k}} \leq 192\right)$, efeito aditivo “fixo" (A) da raça Angus, efeito de dominância Nelore-Angus (D) e efeito epistático aditivo $\times$ aditivo Nelore-Angus (AA). O coeficiente $\mathrm{f}_{1}$ representa a proporção esperada de alelos da raça Angus no l-ésimo animal e $\delta_{l}$ é o coeficiente de heterozigosidade, obtido da probabilidade de que um dos 
alelos tenha derivado da raça Nelore e o outro da raça Angus, para um locus aleatoriamente escolhido de um indivíduo l. Além disso, 2[1-f $\left.\mathrm{f}_{\mathrm{l}}\right] \mathrm{f}_{\mathrm{l}}$ é o coeficiente epistático aditivo $\times$ aditivo para o l-ésimo animal baseado na definição de perdas por recombinação de Kinghorn (1980); u ú o efeito genético aditivo do l-ésimo animal, assumindo que $u=\left\{u_{l}\right\}_{l=1}^{4819} \sim N(0, G)$, em que $G$ é a matriz de covariância genética multirracial, que é uma função das variâncias genéticas específicas por raça, para Angus $\sigma_{\mathrm{g}(1)}^{2}$ e Nelore $\sigma_{\mathrm{g}(2)}^{2}$, a variância de segregação entre as duas raças $\sigma_{s(12)}^{2}$ e o parentesco aditivo entre os animais (Lo et al., 1993); $\mathrm{e}_{\mathrm{ijkl}}$ é o erro residual com distribuição normal homocedástica, isto é $e_{i j k l} \sim N\left(0, \sigma_{e}^{2}\right)$, para todas as combinações de i, j, kel.

Devido à falta de informação objetiva para essa população, a priori, uniformes limitados foram adotados para os efeitos “fixos” $\mu$, sexo $o_{j}$ e coeficientes $\beta^{*}$ 's. Para os efeitos aleatórios de $\mathrm{gc}_{\mathrm{j}}$, u e resíduos foram presumidas distribuições a priori normais conforme descrito acima, contudo especificações conjugadas relativamente não informativas foram adotadas para os componentes de variância, através das seguintes densidades a priori quiquadrado invertidas escalonadas: $\sigma_{g c}^{2} \mid v_{g c}, s_{g c}^{2} \sim s_{g c}^{2} \chi_{g c}^{-2}$; $\sigma_{e}^{2}\left|v_{e}, s_{e}^{2} \sim s_{e}^{2} \chi_{e}^{-2} ; \sigma_{g(r)}^{2}\right| v_{g(r)}, s_{g(r)}^{2} \sim s_{g(r)}^{2} \chi_{g(r)}^{-2}$, em quer=1 para Angus e r=2 para Nelore; e $\sigma_{s(1,2)}^{2} \mid v_{s(1,2)}, s_{s(1,2)}^{2} \sim s_{s(1,2)}^{2} \chi_{s(1,2)}^{-2}$. Neste caso, os hiperparâmetros representados por $v_{g c}, v_{e}$, $v_{g(r)}$ e $v_{s(1,2)}$ definem o grau de confiança nos valores a priori dos componentes de variâncias $\sigma_{g c}^{2}, \sigma_{e}^{2}, \sigma_{g(r)}^{2}$, e $\sigma_{s(12)}^{2}$ dados, respectivamente, pelos hiperparâmetros $s_{g c}^{2}, s_{e}^{2}$, $s_{g(r)}^{2}$, e $s_{s(12)}^{2}$.

Três pressuposições distintas foram adotadas para os componentes de variâncias genéticas específicas de raça e segregação entre raças: 1) Modelo animal multirracial com segregação (MAMRCS): representa o modelo completo apresentado acima, que é composto por duas raças com variâncias genéticas aditivas diferentes e variância de segregação maior que zero; 2) Modelo animal multirracial sem segregação (MAMRSS): representa uma versão simplificada do modelo animal multirracial com segregação, composto por duas raças com variâncias genéticas aditivas diferentes, mas com variância de segregação igual à zero $\left(\sigma_{s(12)}^{2}=0\right)$; 3) Modelo animal (MA): representa o modelo tradicional de avaliação genética, no qual as variâncias genéticas aditivas foram iguais $\left(\sigma_{g(1)}^{2}=\sigma_{g(2)}^{2}\right)$ e a variância de segregação igual à zero $\left(\sigma_{s(12)}^{2}=0\right)$, desse modo foi geneticamente equivalente a uma população de raça pura.

Os valores dos hiperparâmetros foram definidos de forma que a inferência fosse devida essencialmente aos dados, adotando-se graus de confiança baixos, $v_{g(1)}=v_{g(2)}=$ $v_{s(12)}=8 ; v_{g c}=v_{e}=1$, nos valores a priori dos componentes de variância $s_{g(1)}^{2}=s_{g(2)}^{2}=150 \mathrm{~kg}^{2} ; s_{s(12)}^{2}=15 \mathrm{~kg}^{2}$ para modelo animal multirracial com segregação e naturalmente $s_{s(12)}^{2}=0$ para modelo animal e o modelo animal multirracial sem segregação; $s_{g c}^{2}=1000 \mathrm{~kg}^{2}{\text { e } s^{2}}_{e}=350 \mathrm{~kg}^{2}$. Esses valores a priori foram determinados empiricamente com base em análise preliminar dos dados usando modelo animal e máxima verossimilhança residual (dados não apresentados) e em outros da literatura usando o MAMR (Cardoso \& Tempelman, 2004).

A variância genética aditiva de um grupo racial g $\left(\sigma_{\mathrm{g}}^{2}\right)$ formado por duas raças ( $r=1$ e 2) é obtida pela seguinte fórmula (Lo et al., 1993):

$$
\sigma_{g}^{2}=f_{I}^{g} \sigma_{g(l)}^{2}+f_{2}^{g} \sigma_{g(2)}^{2}+2\left(f_{1}^{p} f_{2}^{p}+f_{1}^{m} f_{2}^{m}\right) \sigma_{s(12)}^{2},
$$

em que $f_{r}^{i}$ é a fração da raça r observada no grupo racial $(\mathrm{i}=\mathrm{g})$ e nos grupos paternal $(\mathrm{i}=\mathrm{p})$ e maternal $(\mathrm{i}=\mathrm{m})$. Conseqüentemente, a herdabilidade desse grupo é obtida por:

$$
h_{g}^{2}=\frac{\sigma_{g}^{2}}{\sigma_{g}^{2}+\sigma_{e}^{2}} .
$$

A inferência foi baseada no método Monte Carlo via Cadeias de Markov (MCMC) de 110.000 ciclos, após 10.000 ciclos do período de aquecimento e as amostras foram salvas a cada dez ciclos, para os três modelos, utilizando-se o programa INTERGEN (Cardoso, 2008). As médias, as modas, os percentis $(0,025$ e 0,975$)$ e os desvios-padrão a posteriori dos parâmetros foram obtidos das suas densidades a posteriori marginais. As correlações de ordenamento de Spearman foram calculadas entre os três modelos para as médias a posteriori dos efeitos genéticos aditivos de ganho pós-desmame baseados em todos os animais e touros-pais para todos os indivíduos e para aqueles classificados entre os $10 \%$ superiores.

Para verificar o modelo de melhor ajuste aos dados foram utilizados o critério de informação da deviance e o critério da ordenada preditiva condicional, segundo Gelfand (1996). O critério de informação da deviance é composto por uma medida de ajuste global, a média a posteriori da deviance e uma penalização para a complexidade do modelo. A deviance do modelo $\mathrm{M}_{\mathrm{r}}$ segundo Spiegelhalter et al. (2002) é estimada por $\bar{D}_{r}=\frac{1}{G} \sum_{l=1}^{G}-2 \log p\left(y \mid \theta^{(l)}, M_{r}\right)$, de modo que $\theta^{(l)}$ inclui o valor amostrado para todos os parâmetros do modelo $\mathrm{M}_{\mathrm{r}}$ no ciclo $\mathrm{l}$ de $\mathrm{G}$ ciclos totais. A complexidade do modelo $\mathrm{M}_{\mathrm{r}}$ é determinada pelo número efetivo de parâmetros $p_{\underline{D}(r)}=D^{-}-D_{r}\left(\theta^{-}\right)$, em que $D_{r}\left(\theta^{-}\right)=-2 \log p\left(y \mid \theta^{-}, M_{r}\right)$ com $\theta$ sendo a média a posteriori de $\theta$, isto é, $\mathrm{p}_{\mathrm{D}(\mathrm{r})}$ representa 
a diferença entre a média a posteriori da deviance e a deviance baseada na média a posteriori dos parâmetros sob o modelo $\mathrm{M}_{\mathrm{r}}$. O critério de informação da deviance para o modelo $M_{r}$ é determinado por:

$$
D I C_{r}=\overline{\mathrm{D}}+p_{D(r)} \text {. }
$$

Menores valores de critério de informação da deviance são indicativos de melhor ajuste do modelo aos dados.

Para observação $\mathrm{y}_{\mathrm{ij}}$, a ordenada preditiva condicional é $p\left(y_{i j} \mid y_{(-i j)}, M_{r}\right)$, destinada a ser a densidade de validação cruzada, que sugere quais valores de $y_{i j}$ são prováveis quando o modelo $\mathrm{M}_{\mathrm{r}}$ é ajustado a todas as outras observações em $y_{(-i j)}$ exceto $y_{i j}$ (Gelfand, 1996). Os critérios da ordenada preditiva condicional para o modelo $\mathrm{M}_{\mathrm{r}}$ foram obtidos pela média harmônica de G ciclos de MCMC, por meio da seguinte aproximação:

$$
p\left(y_{i j} \mid y_{(-i j)}, M_{r}\right) \approx \frac{1}{\frac{1}{G} \sum_{l=1}^{G} p^{-1}\left(y_{i j} \mid \theta^{()}, M_{r}\right)} .
$$

Finalmente o estimador da deviance do modelo $r$ baseada no critério da ordenada preditiva condicional é obtido por $-2 \sum_{k=1}^{n} \log \hat{p}\left(y_{k} \mid y_{(k)}, M_{i}\right)$, para a qual os menores valores indicam melhor ajuste.

\section{Resultados e Discussão}

Os critérios adotados para comparar o ajuste dos modelos favoreceram os modelos multirraciais em detrimento ao modelo animal (Tabela 1). Cardoso \& Tempelman (2004) compararam o modelo animal e o modelo animal multirracial com segregação e obtiveram resultados similares, a favor do modelo animal multirracial em estudo com uma população Nelore-Hereford. Na avaliação da importância de incluir a variância de segregação entre raças no modelo multirracial, os resultados desses critérios não foram conclusivos, pois ambos os modelos multirraciais tiveram ajuste similar, com o critério de informação da deviance ligeiramente menor e, portanto, favorável ao modelo com segregação, enquanto a deviance baseada na ordenada preditiva condicional favoreceu aquele sem segregação (Tabela 1). Com esses resultados, o modelo sem segregação, por conter menor número de parâmetros é a alternativa mais recomendável pelo princípio da parcimônia.

A inferência sobre os efeitos genéticos fixos no ganho de peso diário usando a parametrização de perda epistática de Kinghorn (1980) foi similar quando obtida por meio dos três modelos, pois houve sobreposição entre os intervalos formados pela média \pm desvio-padrão a posteriori para todos os parâmetros (Tabela 2). Em todos os modelos, a média do ganho de peso diário descreveu à medida que aumentou a proporção da raça Nelore. Como esperado, a dominância favoreceu o ganho de peso, enquanto a interação aditiva $\times$ aditiva (isto é, perda epistática) teve efeito adverso.

Modelos com maior nível de complexidade não foram considerados, dada a dificuldade de estimar efeitos genéticos fixos não aditivos usando dados de campo, devido a multicolinearidade entre seus coeficientes particularmente quando vários efeitos epistáticos são modelados tais como aditivo $\times$ dominante e dominante $\times$ dominante (Klei \& Quaas, 1996; Birchmeier et al., 2002). Cardoso \& Tempelman (2004) encontraram resultado similar ao comparar o efeito aditivo, de dominância e a interação aditiva $\times$ aditiva entre o modelo animal e o MAMR em estudo com uma população Nelore-Hereford. Outros autores também encontraram valores negativos para epistasia em estudo sobre ganho pré-desmame para uma população multirracial Nelore-Hereford (Carvalheiro et al., 2006; Pimentel et al., 2006).

Houve decréscimo significativo da heterose em cruzamentos avançados (filhos de bimestiços), não somente por causa da diminuição da heterozigose, mas também

Tabela 1 - Média da deviance, penalização por número efetivo de parâmetros (pD), critério de informação da deviance (DIC = Deviance

\begin{tabular}{|c|c|c|c|c|}
\hline Modelo & Deviance & $\mathrm{pD}$ & DIC & Deviance - CPO \\
\hline Modelo animal & 35.271 & 1.156 & 36.427 & 36.672 \\
\hline Modelo animal multirracial com segregação & 35.051 & 1.295 & 36.346 & 36.661 \\
\hline Modelo animal multirracial sem segregação & 35.094 & 1.261 & 36.355 & 36.649 \\
\hline
\end{tabular}
$+\mathrm{pD})$ e deviance ordenada preditiva condicional (Deviance - CPO) calculados para pós-desmama

Tabela 2 - Médias e desvios-padrão a posteriori dos efeitos genéticos fixos para ganho pós-desmama em uma população Nelore-Angus

\begin{tabular}{lcrr}
\hline Efeito (parâmetro) & Modelo animal multirracial sem segregação & Modelo animal multirracial com segregação & Modelo animal \\
\cline { 2 - 4 } & MédiaP \pm DP, kg & MédiaP \pm DP, kg & MédiaP \pm DP, kg \\
\hline Aditivo $\left(\beta_{A}\right)$ & $21,937 \pm 6,7$ & $22,477 \pm 6,7$ & $25,675 \pm 7,8$ \\
Dominância $\left(\beta_{D}\right)$ & $73,076 \pm 4,7$ & $73,145 \pm 4,9$ & $72,484 \pm 5,3$ \\
Aditivo $\times$ aditivo $\left(\beta_{A A}\right)$ & $-63,373 \pm 9,6$ & $-63,223 \pm 9,9$ & $-60,029 \pm 11,4$ \\
\hline
\end{tabular}


devido às perdas por recombinação (Tabela 3; Figura 1). Nas extremidades à direita e à esquerda da figura, observam-se os picos de desempenho para GPD dos animais $\mathrm{F}_{1}$, na extremidade inferior, os animais Nelore puros com pior desempenho e na extremidade oposta (superior), os animais Angus puros com desempenho intermediário, semelhante ao dos animais 3/8 Nelore.

Desempenho similar ao dos animais de cruzamentos avançados com os animais da raça pura que serviu de base materna para o cruzamento (neste estudo o Angus) também foi constatado por outros autores na raça Hereford (Cardoso \& Tempelman, 2004; Carvalheiro et al., 2006). Oliveira et al. (1998) estudaram os pesos ao desmame, ao ano e ao sobreano dos mesmos animais Brangus-Ibagé deste estudo utilizando um modelo que incluiu o efeito de geração e concluíram que os animais da terceira geração tiveram desempenho inferior ao das gerações anteriores. Esses resultados são compatíveis com a diminuição de desempenho observada a partir da terceira geração, pois os efeitos de heterozigose e perdas por recombinação estabilizam de forma global na população, inclusive quanto ao desempenho materno.

As variâncias genéticas obtidas para as raças Nelore e Angus pelo modelo animal multirracial com segregação e pelo modelo animal multirracial sem segregação diferiram substancialmente em magnitude (Tabela 4). A variância genética média a posteriori da raça Angus foi mais que duas vezes maior que a obtida para a raça Nelore com nenhuma sobreposição entre o intervalo de 95\% de probabilidade $a$ posteriori obtido para esses parâmetros (Tabela 4). Usando o modelo animal convencional, uma variância genética média a posteriori intermediária entre as médias a posteriori da raça Nelore e da raça Angus nos modelos animais multirraciais com segregação e sem segregação foi obtida, porém essa variância genética comum a todas as composições raciais foi mais próxima da observada para a raça Angus, conforme observado nas densidades a posteriori desses componentes de variância (Figura 2). Esse resultado pode ser atribuído à maior proporção esperada de genes Angus na população, já que a composição racial média da população é de 63,14\% Angus. Cardoso \& Tempelman (2004) encontraram variância para a raça Nelore de 27,9\% da magnitude da raça Hereford para ganho de peso diário, enquanto a raça Nelore teve variância genética de 43,4\% para essa característica em relação à raça Angus. A média a posteriori da variância devida à segregação entre as raças Angus e Nelore foi de aproximadamente 12,9\% da variância genética média a posteriori da raça Nelore, contudo representou somente 5,6\% da variância genética média a posteriori da raça Angus (Tabela 4). A magnitude da variância da segregação relativa à variância genética da raça Angus (5,6\%) foi, entretanto, menor que a de 16,5\% obtida em relação à raça Hereford para peso ao nascer de cruzamentos de Hereford-Nelore na Argentina (16,5\%) (Birchmeier et al., 2002), de 26,8\% em relação ao Charolês para peso ao desmame de cruzamentos de Charolês-Zebu

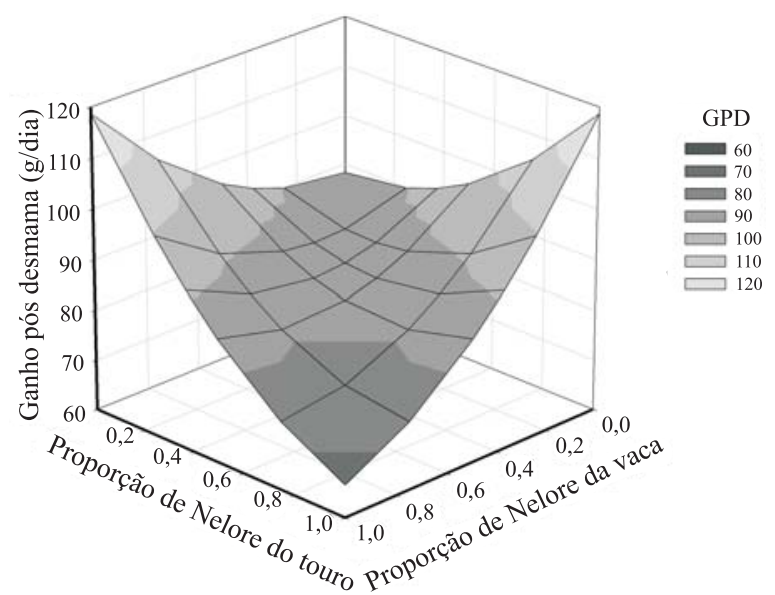

Figura 1 - Ganho pós-desmama estimado pelo modelo animal multirracial sem segregação (MAMRSS) para animais Angus-Nelore de acordo com a proporção de Nelore.

Tabela 3 - Distribuição de registros, número total de indivíduos com registros, coeficientes genéticos ${ }^{1}$ e ganho médio pós-desmama por grupo de composição racial² em conjunto de dados Nelore (N) - Angus (A) para ganho pós-desmama

\begin{tabular}{|c|c|c|c|c|c|c|c|c|}
\hline Composição racial & Nelore & $\begin{array}{c}\text { Média } \\
\text { observada, kg }\end{array}$ & $\mathrm{f}_{l}$ & $\delta_{l}$ & $2\left[1-\mathrm{f}_{l}\right] \mathrm{f}_{l}$ & $\begin{array}{c}\text { Média ajustada, } \\
\text { kg (MA) }\end{array}$ & $\begin{array}{l}\text { Média ajustada, } \\
\text { kg (MAMRCS) }\end{array}$ & $\begin{array}{l}\text { Média ajustada, } \\
\text { kg (MAMRSS) }\end{array}$ \\
\hline Angus & 385 & $84,5 \pm 38,34$ & 1,0000 & 0,0000 & 0,0000 & $88,2 \pm 2,74$ & $88,2 \pm 2,74$ & $88,2 \pm 2,78$ \\
\hline$(1 / 2 N-1 / 2 A) F 1$ & 174 & $128,6 \pm 41,68$ & 0,5000 & 1,0000 & 0,5000 & $117,9 \pm 3,35$ & $118,5 \pm 3,04$ & $118,6 \pm 3,08$ \\
\hline$(3 / 4 \mathrm{~N}-1 / 4 \mathrm{~A})$ & 313 & $98,7 \pm 34,98$ & 0,2500 & 0,5000 & 0,3750 & $82,7 \pm 4,13$ & $84,2 \pm 3,63$ & $84,5 \pm 3,66$ \\
\hline$(3 / 4 \mathrm{~A}-1 / 4 \mathrm{~N})$ & 71 & $94,0 \pm 31,65$ & 0,7500 & 0,5000 & 0,3750 & $95,5 \pm 2,14$ & $95,5 \pm 2,11$ & $95,5 \pm 2,09$ \\
\hline$(3 / 4 \mathrm{~A}-1 / 4 \mathrm{~N})$ avançado & 92 & $40,4 \pm 44,12$ & 0,7500 & 0,3750 & 0,3750 & $86,5 \pm 2,16$ & $86,3 \pm 2,14$ & $86,3 \pm 2,06$ \\
\hline$(3 / 8 \mathrm{~N}-5 / 8 \mathrm{~A}) \mathrm{PZ} / \mathrm{ZP}$ & 317 & $88,4 \pm 49,08$ & 0,6250 & 0,7500 & 0,4687 & $104,8 \pm 2,34$ & $105,0 \pm 2,26$ & $105,1 \pm 2,28$ \\
\hline$(3 / 8 \mathrm{~N}-5 / 8 \mathrm{~A}) \mathrm{ZA} / \mathrm{AZ}$ & 350 & $64,7 \pm 53,52$ & 0,6250 & 0,5000 & 0,4687 & $86,7 \pm 2,09$ & $86,7 \pm 2,08$ & $86,8 \pm 2,02$ \\
\hline$(3 / 8 \mathrm{~N}-5 / 8 \mathrm{~A}) \mathrm{PA}$ & 2314 & $96,4 \pm 38,44$ & 0,6250 & 0,4687 & 0,4687 & $84,4 \pm 2,12$ & $84,5 \pm 2,11$ & $84,6 \pm 2,03$ \\
\hline
\end{tabular}

${ }^{1} f_{l}$ representa à proporção esperada de alelos da raça Angus; $\delta_{l}$ é o coeficiente de heterozigosidade Nelore-Angus e $2\left[1-f_{l}\right] f_{l}$ é o coeficiente epistático aditivo $\times$ aditivo ou de perdas por recombinação de Kinghorn (1980).

2 Grupo racial (composição do touro vs. composição da vaca): PZ (A vs. 3/4 N - 1/4 A); ZP (3/4N - 1/4A vs. Angus); ZA (1/2N - 1/2A × 1/4N - 3/4A); AZ (1/4N - 3/4A × 1/2N - 1/2A); PA (PZ, ZP, ZA, AZ vs. PZ, ZP, ZA, AZ); MA = modelo animal; MAMRCS = modelo animal multirracial com segregação; MAMRSS = modelo animal multirracial sem segregação. 
Tabela 4 - Médias e desvio-padrão a posteriori, moda a posteriori, intervalo de 95\% de probabilidade $a$ posteriori e tamanho efetivo das amostras ${ }^{1}$ para as variâncias médias de uma população Brangus-Ibagé

\begin{tabular}{|c|c|c|c|c|c|}
\hline Variâncias & $\begin{array}{l}\text { Médias } a \\
\text { posteriori }\end{array}$ & $\begin{array}{l}\text { Desvio-padrão } \\
\text { a posteriori }\end{array}$ & $\begin{array}{c}\text { Moda } a \\
\text { posteriori }\end{array}$ & $\begin{array}{c}\text { Intervalo de } 95 \% \\
\text { de probabilidade a posteriori }\end{array}$ & $\begin{array}{c}\text { Tamanho efetivo } \\
\text { das amostras }\end{array}$ \\
\hline \multicolumn{6}{|l|}{ Modelo animal } \\
\hline Genética $^{2}$ & 152,84 & 19,61 & 150,54 & 116,75 a 193,47 & 946 \\
\hline \multicolumn{6}{|c|}{ Modelo animal multirracial com segregação } \\
\hline Angus & 189,06 & 26,12 & 183,18 & 140,61 a 241,75 & 927 \\
\hline Nelore & 82,01 & 22,95 & 74,34 & 45,91 a 135,10 & 1655 \\
\hline Residual & 361,83 & 17,46 & 363,92 & 327,59 а 395,59 & 1332 \\
\hline \multicolumn{6}{|c|}{ Modelo animal multirracial sem segregação } \\
\hline Angus & 189,76 & 27,06 & 192,28 & 140,19 a 244,49 & 819 \\
\hline Nelore & 83,58 & 23,33 & 75,92 & 45,88 а 136,50 & 2115 \\
\hline PA $(3 / 8 N-5 / 8 A)^{3}$ & 149,95 & 17,43 & 149,94 & 117,37 a 185,37 & 893 \\
\hline Residual & 365,87 & 17,70 & 365,71 & 331,60 a 400,82 & 1226 \\
\hline
\end{tabular}

${ }^{1}$ Conforme proposto por Sorensen et al. (1995); ${ }^{2}$ Comum a todos os grupos raciais; ${ }^{3} \mathrm{PA}(3 / 8 \mathrm{~N}-5 / 8$ A): puro sintético avançado.
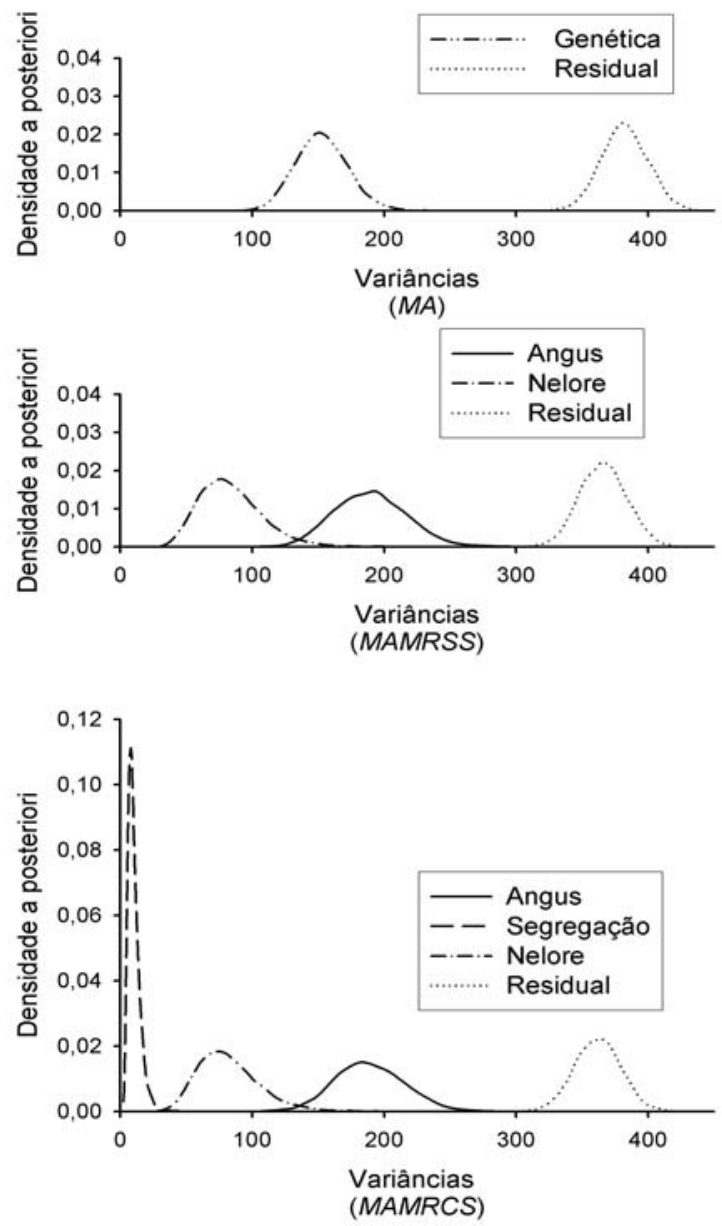

Figura 2 - Densidades a posteriori da variância genética aditiva e da variância residual do modelo animal (MA); densidades a posteriori das variâncias genéticas aditivas raciais e da variância residual do modelo animal multirracial sem segregação (MAMRSS) e as densidades a posteriori das variâncias genéticas aditivas raciais e da segregação do modelo animal multirracial (MAMRCS) e da variância residual para ganho pós-desmame em Nelore-Angus. no Brasil (Toral, 2007), de 6,3\% para peso ao desmame de cruzamentos de Angus-Brahman nos Estados Unidos (Elzo \& Wakeman, 1998) e de 9,9\% para ganho de peso diário de Hereford-Nelore no Brasil (Cardoso \& Tempelman, 2004). Essa menor magnitude da variância da segregação neste estudo explica, ao menos parcialmente, o ajuste similar dos modelos multirraciais com e sem segregação (Tabela 1). Houve uma variação muito pequena nas variâncias genéticas raciais entre esses modelos quando a variância da segregação foi fixada em zero no modelo animal multirracial sem segregação e esse componente foi absorvido pela variância residual desse modelo (Tabela 4; Figura 2). Além disso, a densidade de valores da variância da segregação no modelo animal multirracial com segregação está concentrada próximo a zero (Figura 2), resultados que sugerem que uma avaliação genética multirracial da população estudada pode ser satisfatoriamente implementada por meio de um modelo sem variância da segregação. Por sua vez, a herdabilidade do GPD estimada para os animais puros sintéticos avançados da presente população experimental, de 0,29 , foi superior à encontrada para ganho diário médio pós-desmama de animais da raça Brangus, participantes do programa Natura Genética Sul Americana e criados nas regiões Sul, Sudeste e Centro-Oeste do Brasil, que foi de 0,20 (Lopes et al., 2009).

A existência de um efeito escalar nas variâncias fenotípicas, observado quando grupos com média fenotípica maior têm também variância fenotípica maior (Falconer \& Mackay, 1996), poderia acentuar a diferença entre as variâncias genéticas das raças Angus e Nelore, dada a pressuposição de variância residual homogênea neste estudo. Entretanto, não há evidências de efeito escalar nos dados (Tabela 3) e não se constatou nenhuma relação 
entre a média observada e os desvios-padrão fenotípicos, que foram bastante similares entre os diferentes grupos genéticos. Por outro lado, a validade da pressuposição de variância residual homocedástica pode ser testada utilizando modelos estruturais heterocedásticos (Foulley et al., 1992), obtido pela adição de estágios extras ao modelo multirracial, conforme proposto por Cardoso et al. (2005).

As correlações de ordenamento de Spearman entre os valores genéticos obtidos pelos modelos foram calculadas considerando todos os animais avaliados e incluindo somente os touros pais, os $10 \%$ superiores em ganho de peso diário de todos os animais e os $10 \%$ superiores aos touros pais em ganho de peso diário (Tabela 5). As correlações de Spearman foram maiores entre os valores genéticos quando considerados todos os animais em relação à subamostra contendo os $10 \%$ superiores para todos os modelos. Essas correlações de ordem entre os modelos multirraciais sem segregação e com segregação foram próximas de 1 em todos os grupos animais considerados, o que indica que o ordenamento dos animais para a seleção é praticamente o mesmo pelos dois modelos. Por outro lado, foram observadas diferenças entre o modelo animal convencional e os modelos multirraciais com e sem segregação quando selecionados animais superiores como reprodutores (Tabela 5). A magnitude observada neste estudo quando considerados apenas os $10 \%$ superiores foi similar à obtida por Cardoso \& Tempelman (2004), de 0,875, para a correlação de ordenamento de Kendall entre modelo animal e modelo animal multirracial para todos os animais de uma população cruzada Nelore-Hereford, e isso comprova que os animais selecionados considerando de forma apropriada variâncias genéticas multirraciais não são necessariamente os mesmos quando selecionados usandose o modelo animal.

Tabela 5 - Correlação de ordem (Spearman) ${ }^{1}$ entre valores genéticos individuais de todos os touros pais e todos os animais

\begin{tabular}{|c|c|c|c|}
\hline & Modelo animal & $\begin{array}{l}\text { Modelo animal multirracial } \\
\text { com segregação }\end{array}$ & $\begin{array}{l}\text { Modelo animal multirracial } \\
\text { sem segregação }\end{array}$ \\
\hline \multicolumn{4}{|l|}{ Touros pais } \\
\hline Modelo animal & & 0,995 & 0,996 \\
\hline Modelo animal multirracial com segregação & 0,830 & & 0,999 \\
\hline Modelo animal multirracial sem segregação & 0,818 & 0,988 & \\
\hline \multicolumn{4}{|l|}{ Todos os animais } \\
\hline Modelo animal & & 0,993 & 0,993 \\
\hline Modelo animal multirracial com segregação & 0,910 & & 0,999 \\
\hline Modelo animal multirracial sem segregacão & 0,916 & 0,998 & \\
\hline
\end{tabular}

${ }^{1}$ Diagonal inferior $10 \%$ superiores.

\section{Conclusões}

A heterogeneidade de variância genética constatada entre os grupos genéticos do rebanho Brangus-Ibagé estudado é essencialmente devida à diferença entre as variâncias das raças Angus e Nelore. Consequentemente, um modelo multirracial que não inclua a variância devida à segregação entre essas duas raças é uma alternativa parcimoniosa adequada para avaliação genética multirracial dessa população. Para aumentar o ganho pós-desmama, deve-se favorecer os grupos genéticos com maior dominância e menor epistasia aditiva $\times$ aditiva. Alterações na classificação de ordem dos animais candidatos à seleção entre o modelo animal tradicionalmente usado nas avaliações genéticas e modelos animais multirraciais que apresentaram melhor ajuste aos dados de ganho pós-desmama indicam que esses modelos multirraciais, devido à decomposição da variância genética aditiva nos seus componentes raciais, permitem a predição de valores genéticos mais acurados e podem melhorar o progresso genético das populações formadas a partir do cruzamento entre duas ou mais raças.

\section{Agradecimentos}

Ao Dr. Nelson Manzoni de Oliveira da Embrapa Pecuária Sul, pelo auxílio na estruturação do banco de dados utilizados neste trabalho. Ao CNPq - Conselho Nacional de Desenvolvimento Científico e Tecnológico, pela concessão da bolsa de estudos.

\section{Referências}

ARNOLD, J.W.; BERTRAND, J.K.; BENYSHEK, L.L. Animal model for genetic evaluation of multibreed data. Journal of Animal Science, v.70, n.11, p.3322-3332, 1992.

BIRCHMEIER, A.N.; CANTET, R.J.C.; FERNANDO, R.L. et al. Estimation of segregation variance for birth weight in beef cattle. Livestock Production Science, v.76, n.1-2, p.27-35, 2002. 
CARDOSO, F.F. Manual de utilização do Programa INTERGEN - Versão 1.0 em estudos de genética quantitativa animal. Bagé: Embrapa Pecuária Sul, 2008. p.74. (Documentos, 74).

CARDOSO, F.F.; ROSA, G.J.M.; TEMPELMAN, R.J. et al. Multiple-breed genetic inference using heavy-tailed structural models for heterogeneous residual variances. Journal of Animal Science, v.83, n.8, p.1766-1779, 2005.

CARDOSO, F.F.; TEMPELMAN, R.J. Hierarchical Bayes multiplebreed inference with an application to genetic evaluation of a Nelore-Hereford population. Journal of Animal Science, v.82, n.6, p.1589-1601, 2004.

CARVALHEIRO, R.; PIMENTEL, E.C.G. et al. Genetic effects on preweaning weight gain of Nelore-Hereford calves according to different models and estimation methods. Journal of Animal Science, v.84, n.11, p.2925-2933, 2006.

ELZO, M.A. Restricted maximum likelihood procedures for the estimation of additive and nonadditive genetic variances and covariances in multibreed populations. Journal Animal Science, v.72, n.12, p.3055-3065, 1994.

ELZO, M.A.; WAKEMAN, D.L. Covariance components and prediction for additive and nonadditive preweaning growth genetic effects in an Angus-Brahman multibreed herd. Journal of Animal Science, v.76, n.5, p.1290-302, 1998.

FALCONER, D.S.; MACKAY, T.F.C. Introduction to quantitative genetics. Harlow: Longman Group Ltda. 1996. 464p.

FERNANDO, R.L. Theory for analysis of multi-breed data. In: GENETIC PREDICTION WORKSHOP, 7., 1999, Kansas City. Proceedings... Kansas City: Beef Improvement Federation, 1999. p.1-16.

FOULLEY, J.L.; CRISTOBAL, M.S.; GIANOLA, D. et al. Marginal likelihood and Bayesian approaches to the analysis of heterogeneous residual variances in mixed linear Gaussian models. Computational Statistics \& Data Analysis, v.13, n.3, p.291-305, 1992.

GELFAND, A.E. Model determination using sampling-based methods. In: GILKS, W.R.; RICHARDSON, S.; SPIEGELhalter, D.J. (Eds.). Markov Chain Monte Carlo in practice. London: Champman \& Hall, 1996. p.145-161.

GREGORY, K.E.; CUNDIFF, L.V. et al. Composite breeds to use heterosis and breed differences to improve efficiency of beef production. Clay Center: MARC-USDAARS, 1999. 75p.
KINGHORN, B. The expression of recombination loss in quantitative traits. Zeitschrift Fur Tierzuchtung Und Zuchtungsbiologie-Journal of Animal Breeding and Genetics, v.97, n.2, p.138-143, 1980.

KLEI, L.; QUAAS, R.L.; POLLAK, E.J. Multiple-breed evaluation. In: BEEF IMPROVEMENT FEDERATION RESEARCH SYMPOSIUM \& ANNUAL MEETING, 28., 1996, Birmingham. Proceedings... Birmingham, 1996. p.93-105.

LO, L.L.; FERNANDO, R.L.; GROSSMAN, M. Covariance between relatives in multibreed populations: additive model. Theoretical and Applied Genetics, v.87, n.4, p.423-430, 1993.

LOPES, J.S.; RORATO, P.R.N.; WEBER, T. et al. Parâmetros genéticos e tendências genética e fenotípica para características de crescimento em uma população da raça Brangus. Revista Brasileira de Zootecnia, v.38, n.4, p.662-669, 2009.

OLIVEIRA, N.M.; SALOMONI, E.; LEAL, J.J.B. et al. Genetic and environmental effects on growth of $3 / 4$ Nelore $x$ 5/8 Aberdeen Angus beef cattle derived from different crossbreeding schemes. Archivos Latinoamericanos de Producción Animal, v.6, n.2, p.173-188, 1998.

PIMENTEL, E.D.C.G.; QUEIROZ, S.A.D.; CARVALHEIRO, R. et al. Estimativas de efeitos genéticos em bezerros cruzados por diferentes modelos e métodos de estimação. Revista Brasileira de Zootecnia, v.35, n.3, p.1020-1027, 2006.

ROSO, V.M.; SCHENKEL, F.S. AMC - A computer program to assess the degree of connectedness among contemporary groups. In: WORLD CONGRESS ON GENETICS APPLIED TO LIVESTOCK PRODUCTION, 8., 2006, Belo Horizonte. Proceedings... Belo Horizonte, 2006. p.26. (Communication, 27).

SALOMONI, E.; CHAGAS, E.C.; DEL DUCA, L.O.A. Ibagé: formação e seleção de uma nova raça. Bagé: EMBRAPAUEPAE de Bagé, 1984. 23p. (Circular Técnica, 1).

SORENSEN, D.A.; ANDERSEN, S.; GIANOLA, D. et al. Bayesianinference in threshold models using Gibbs sampling. Genetics Selection Evolution, v.27, n.3, p.229-249, 1995.

SPIEGELHALTER, D.J.; BEST, N.G.; CARLIN, B.P. et al. Bayesian measures of model complexity and fit. Journal of the Royal Statistical Society Series B-Statistical Methodology, v.64, p.583-616, 2002.

TORAL, F.L.B. Modelos para avaliação do peso à desmama de bovinos cruzados Charolês-Zebu. 2007. 138f. Tese (Doutorado em Zootecnia) - Universidade Federal de Viçosa, Viçosa, MG. 